\title{
Smart Augmented Learning
}

\author{
Shanthini.E ${ }^{\mathrm{a}}$, Sangeetha. $V^{\mathrm{b}}$, Siva Raam.Mc ${ }^{\mathrm{c}}$,SreeDhviya.M ${ }^{\mathrm{d}}$, VarshiniMeenaskhi.J ${ }^{\mathrm{e}}$ and Venkatesh.C.S ${ }^{\mathrm{f}}$ \\ A \\ Assistant Professor, Department of Electronics and Communication Engineering, \\ Sri Ramakrishna Engineering College, Coimbatore, 641041, Tamilnadu,India \\ bAssistant Professor, Department of Electronics and Communication Engineering, \\ Sri Ramakrishna Engineering College, Coimbatore, 641041, Tamilnadu,India

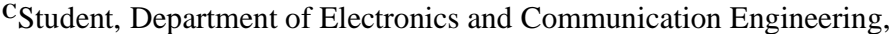 \\ Sri Ramakrishna Engineering College, Coimbatore, 641041, Tamilnadu,India \\ dStudent, Department of Electronics and Communication Engineering, \\ Sri Ramakrishna Engineering College, Coimbatore, 641041, Tamilnadu,India \\ eStudent, Department of Electronics and Communication Engineering, \\ Sri Ramakrishna Engineering College, Coimbatore, 641041, Tamilnadu,India \\ fStudent, Department of Electronics and Communication Engineering, \\ Sri Ramakrishna Engineering College, Coimbatore, 641041, Tamilnadu,India
}

Article History: Received: 11 January 2021; Accepted: 27 February 2021; Published online: 5 April 2021

\begin{abstract}
Education is the foundation of our society. The most important investment we can make collectively and individually is in education. Rural education is of utmost importance to enhance and develop the livelihood of rural people indirectly. But the face of education in rural parts of the country has to be considered for a serious check-up with children failing to receive a good quality of education. This is one of the reasons to drivestudents away from attending their schools. The education system in rural areas is information based rather than a practical oriented, which is a major setback for the economic growth of the nation. The prominent crises that hold back rural schools are the lack of smart learning technology and no proper access to laboratory equipment for the students. Augmented Reality (AR) is one of the finest technologies that can be utilized for education development as it amplifies the learning process.Marker-based AR android application can help by over-laying audiovisuals, computer graphics, 3-D graphical models' representation, the contextual information of concept's procedures, processes, working, functions and its usage directly over the manuals which make the subject more understandable and comprehensive, thus enhances the student's knowledge. The utilization of AR technology in the field of education will prove to be a great positive societal impact essentially for rural school students. It sets a path for learning all the concepts smartly and efficiently. AR-based learning can create an impact because there is no need for the internet. The mobile application also helps to improve the student's self-learning skills. In this paper, some concepts from biology and physics are considered, the graphical models, functionality and working are displayed in AR with the help of Maya 3D, Unity 3D.
\end{abstract}

Keywords: Augmented Reality, 3-D Visualization, Immersive experience, Marker-based Augmented Reality, Smart education, AR-based Laboratory.

\section{Introduction}

A great number of Indians still live in rural areas. In India, as per 8th AISES done by ESD, NCERT report[7], out of 82468 secondary schools in the rural area; only 36,121 (43.30\%) have computers which are used for teaching and learning purpose and the remaining $57 \%$ of the rural schools lack lab equipment and facilities. They also lack knowledge on current and upcoming technologies. Modern-day education system requires the students to be aware of what they are learning and also how they apply that in the real world. This can be done only when basic concepts are clear for the students right from schooling. A study [9] shows that a village school in Uttaranchal, Pithoragarh district does not have proper educational facilities. The rural school students are not able to handle the laboratory equipment because of the lack of facilities. The days where education was only with books, blackboard and chalk are gone. The urban children get to learn from smartboards and high-end electronic gadgets which is not possible for rural children. This also stands as a setback for rural school children. Nowadays, with more technology getting emerged, we can utilize them for the growth of the younger generation. AR smart laboratory is designed for the students to get practical knowledge. This technology works by enhancing the current perception of reality. In contrast to virtual reality (VR), AR augments computer-generated graphics over the real world. In most academic setup, teachers can provide students with only limited opportunities because of repeat exposure to the same information. Here AR-based approach will effectively work. Marker-based AR is an android application that allows the user to scan physical objects and render a 3D model to interact with it using the device. Some of the existing methods are of 2D contents whereas the app is completely 3D and more appealing.

Yang Kuang and Xiaomi Mei work show their analysis on the pre-school children's psychological characteristics[1]. The thinking ability of the children gets improvised via AR learning methods. As it is an advanced technology, it allows learning through remote collaboration (i.e.) the teachers and students may not 
necessarily be present in the same location but can share a similar virtual environment for learning with digital computer-generated objects and students have the access to interact within that setting. The mobile AR offers a vast opportunity for educators to change the way of teaching and for students to implement a different learning strategy. Google's Android and Apple's iOS provides good technical support for AR mobile app design development.

To develop the learning content that involves or emphasizes the steps and sequences, acquisition of procedural knowledge is essential and the significance of AR acquisition is vital. Here the traditional way of learning is compared with AR-based modern learning in terms of memory retaining ability.[2] A study on 68 participants had revealed that AR helped the students retain adequate memory of procedural operations even 2 weeks after the completion of classes.

The employment of optical character recognization technology in conjunction with SVSS[2] (Streamlined Viewport Strategy System) and used in holographic AR engine. SVSS can recognize the interfaces, contextual data, relative position of windows on the computer screen as well as augment instructions. This would automatically guide the students to proceed with the subsequent tasks. They could also repeat a task if they face any difficulty in understanding the concepts. This helps learners complete their learning goals.

Recently AR is used for learning Astronomical concepts[3].Mobile Augmented Reality application is an educational gamified approach that intends to teach about astronomical planetary concepts to the primary school students in both formal and informal learning environments. The system relies on GPS, gyroscope and accelerometer devices present in modern smartphones. Thus, the students can learn the astronomical concepts at an early age and acquire knowledge about the solar system with a clear picture. Also, they can have a real-time visualization through MAR app. Technical difficulties such as GPS instability incorrect gyroscope reading can seriously limit user experience.

In 2012, simulation teaching in 3D AR environment[4] was developed to devise several simulations for basic physics concepts like Newton's law, simple pendulum, ant's meeting algorithm, etc., Such learning enhances students interest towards AR-based learning. [5] In 2019, card-based interaction to design visualizations in AR environment was developed by a group of researchers. This development was started using unity gaming engine for user interface along with Vuforia. This comprises an engine module, InfoVis task, visualization module, development environment. The card database consists of visualization card, function card and interaction card. The remark to be noticed is that the filter feature needs to be carefully implemented because it is vital to use in the visualization process. It also presents a lot of challenges in tasks related to selection precisely as it is an interaction involving both real and virtual objects.[6]Kaufmann realizes the interaction between teachers and the students with the help of AR. This system can present the structure of simple points, lines, sides and possesses the function of Boolean calculation.

\section{Materials and Methods}

\subsection{Proposed work}

The technology in the proposed solution is AR which also uses AI for image recognition. The proposed solution was developed using the software Autodesk Maya, Unity 3D. The solution aims at improving the practical knowledge of the students. Learning concepts can be visualized and also students can interact with the generated AR models. Thus, students can understand the concepts in a better manner. This paper presents an educational AR android application such that it will let the students acquire more practical knowledge of biology, physics and also current technology like robotics[10][11]. This method can be significantly beneficial to the students and seems to be cost-effective for rural schools as they don't have to buy all the equipment.AR is a technology that blend computer-generated imagery and AR information about the surrounding real world of the user becomes interactive and dignitary usable. The AR environment provides users with seamless environments to interface the real world and the virtual world.

The Maya 3D is used to design the 3D models. Various UI and information are incorporated for the 3D objects by using Unity 3D software. Finally, the android application is developed for use. It is a visualization process; students can convert procedural memory into a long-term memory which is an advanced approach. AspertheNationalEducationPolicy2019[8],theIndian governmenthastakeninitiativesbyprovidingtabletsforelearning andsupportingnewtechnologies. From "Hindustan-Times"[9]dated onSeptember4th,2019, tabletshavebeenprovidedto a fewschools locatedatHardaunvillage (Uttar Pradesh).With thehelpofthe tabletsprovided bythegovernment,students can have access to smartAR android application. Also, the image Target can be downloaded and used as required or the experiment book provided can also be used. The beneficiaries of this work would be the rural school students, teachers/staff, as well as the technicians.

\subsection{Description}

The cameras that are built in the AR-enabled devices are used for environmental detection, depth calculation and to track down the image target. Marker-based AR android application is designed, that allows the user to scan physical objects and render a 3D model to interact with it using the device. Markers are image targets. Here we use the experiment book as the Image Target. The application consists of the list of experiments according to the subject. Once the required experiment page is scanned using the camera in the app the 3D model along with contextual information is provided. An added-up advantage is an audio aid where the information is provided as audio also. It is very easy to learn about the app, and it is very useful to learn through the app. 


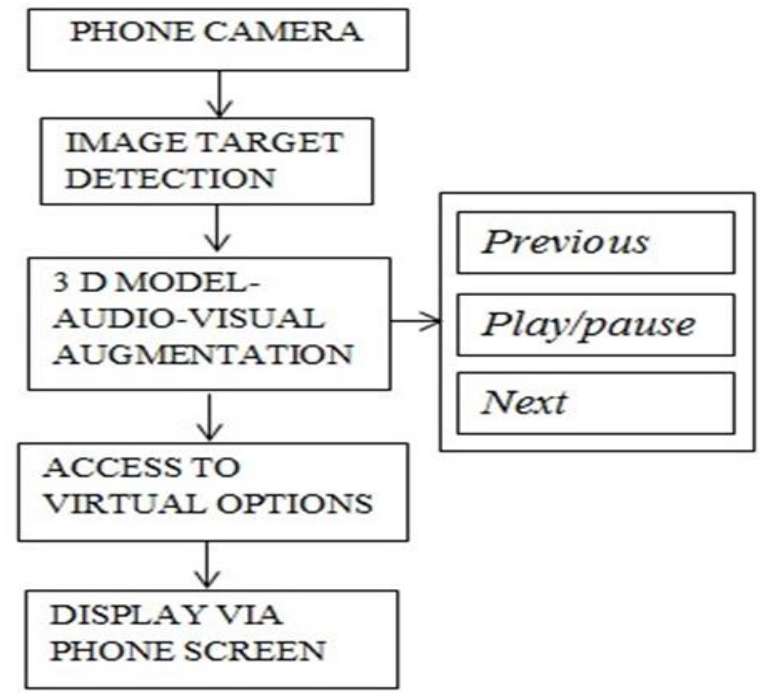

Figure 1 Front -end process of the application

For example, to visualize physical laboratory devices, anatomical structures of human and plants that would be useful for biology students. Students can efficiently learn the concepts and procedures within a very short time and easily understand their applications as well. Also, another example Even without having a robotic arm in place and with only the picture of a robotic arm the full study of the robot arm can be done by the students as this AR application provides adequate information of how robotics evolved and how a robot works also its operation and functions. Figure 1 represents the entire front-end process. This application provides adequate information on how robotics (i.e.) current technology and also laboratory experiment related to biology and physics the 3D graphical representation is projected in front of the user rendering augmented scenes. The provision of audio aid and user interface button makes the application more user friendly, feasible and interactive.Figure 2 shows the backend work.The software tools used are Unity 3D and Maya 3D. Here, the memory unit consists of the database of the application.

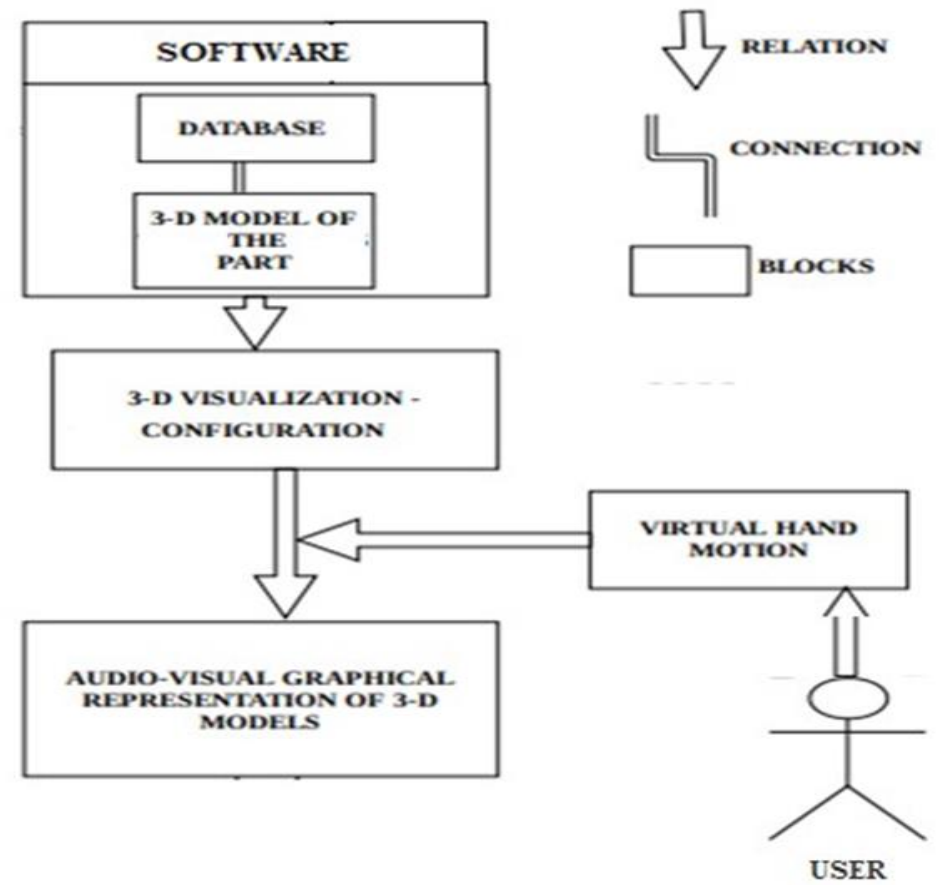

Figure 2 Back-end process

\subsection{Designing and Modelling}

\subsubsection{Maya}

It is a 3-D software application that enables the creation of three-dimensional structures of objects. In the proposed solution, the use of Maya has resulted in the creation of individual parts of a robotic arm in 3D with greater precision. To develop a complete 3D model, some of the significant tools like move, rotate, create a polygon, scaling, rigging, sculpting tools are utilized. Figure 3 depicts the developed robotic arm 3D model in the Maya software. The application aims at delivering visual information features, function and operation are effectively defined. This approach is applicable for any other picture of 3D models equipment. 


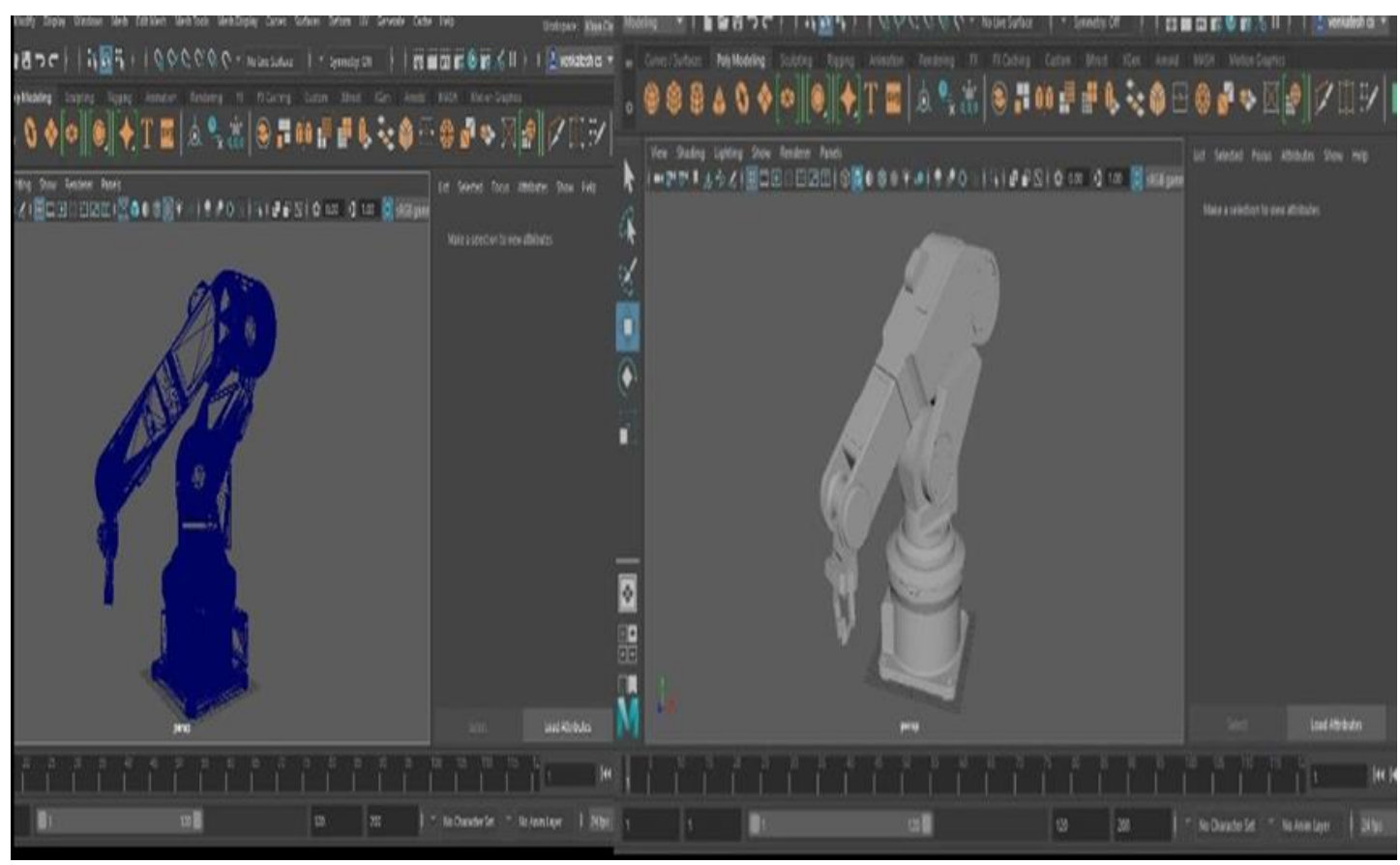

\subsubsection{Unity}

Figure 3 3-D designing and modelling using the Maya software tool

Unity 3D is a platform that supports AR. Unity 3D is the software using which the AR part is done for this application. The 3D model that is designed from MAYA is brought into unity and the contextual information and audio aid are provided in Unity. The UI work, simulation is carried out in unity with C\# code.

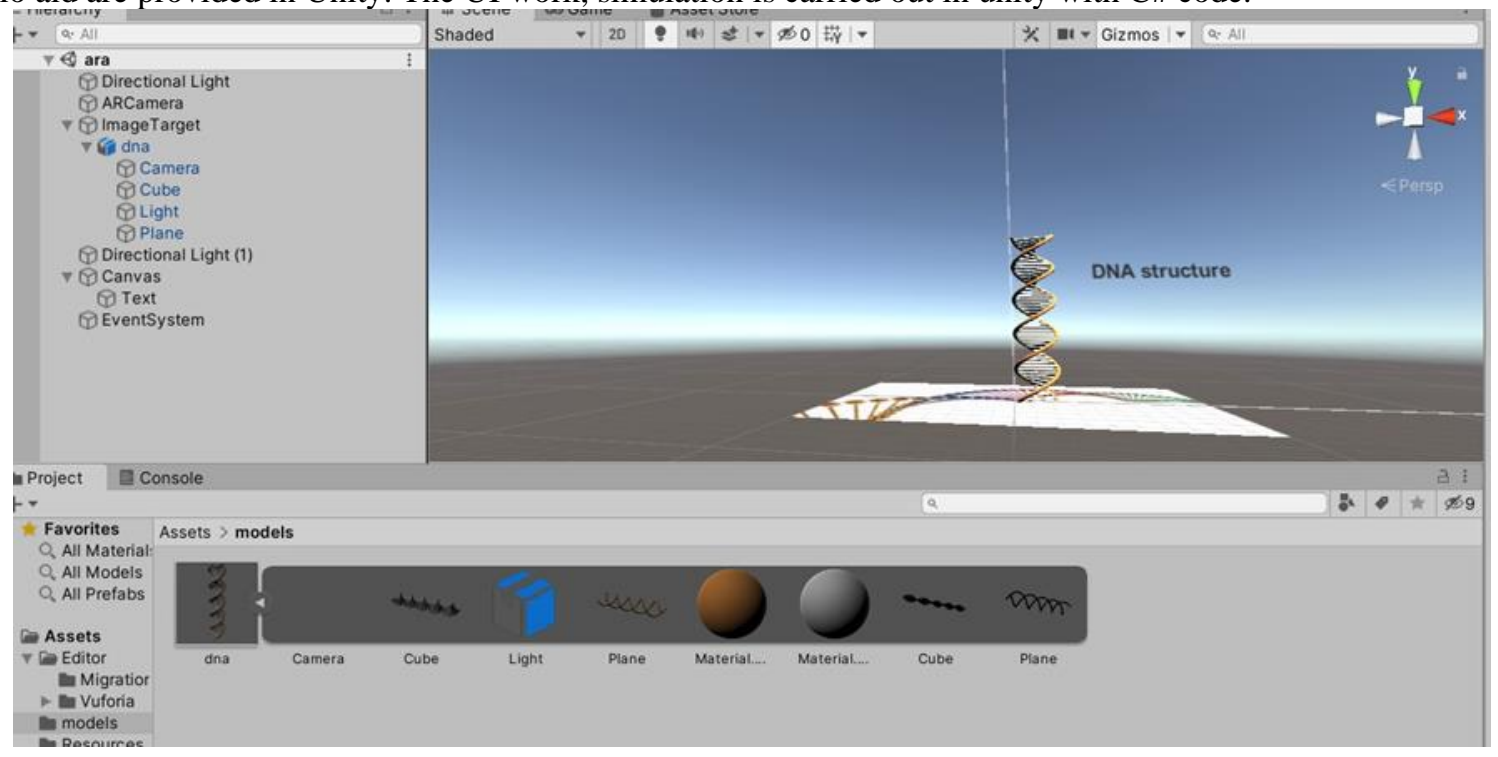

Figure 4 Worksetup in the Unity software tool

Using android studio and Vuforia in unity the work is converted into an android application. In figure 4 the work setup in Unity software tool is shown.The application is intended for android users with an android version of 7.0 and above only.

\subsubsection{Vuforia}

Vuforia Engine is a plugin that is used in Unity 3D to develop the smart Augmented Reality education applications. Figure 5 represents the image registration capability of Vuforia is useful for marker-based AR. It is used to position and orient virtual objects (3D models, User Interfaces and also the contextual information) as a part of the user's real-world scene. 


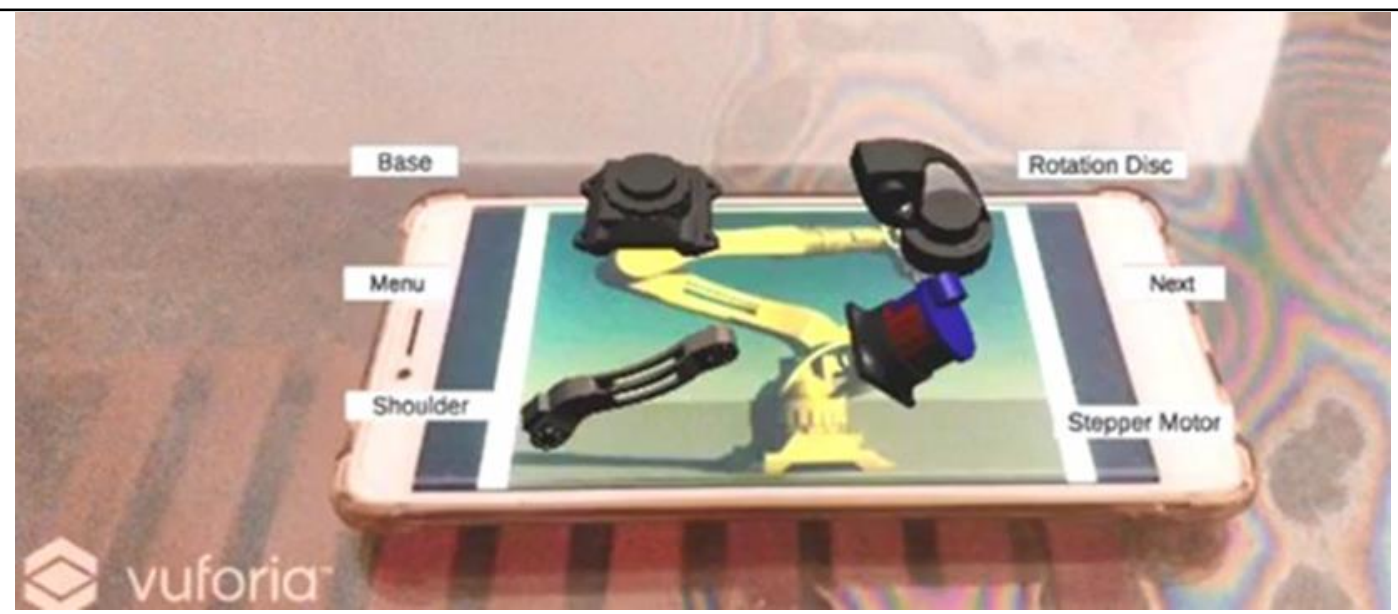

Figure 5 Viewer's perspective

They are viewed through the camera placed in the device, using which the smart education application is viewed. The virtual objects track the position and orientation of the image target in usersscene so that the users' perspective on the object corresponds with the perspective on the image target. The users can interact with the smart education application using Virtual Interface.

\section{Result}

The representation of audiovisual 3D graphical models over the image targethelps the studentto understand the concepts and develop their practical knowledge in the best way possible.

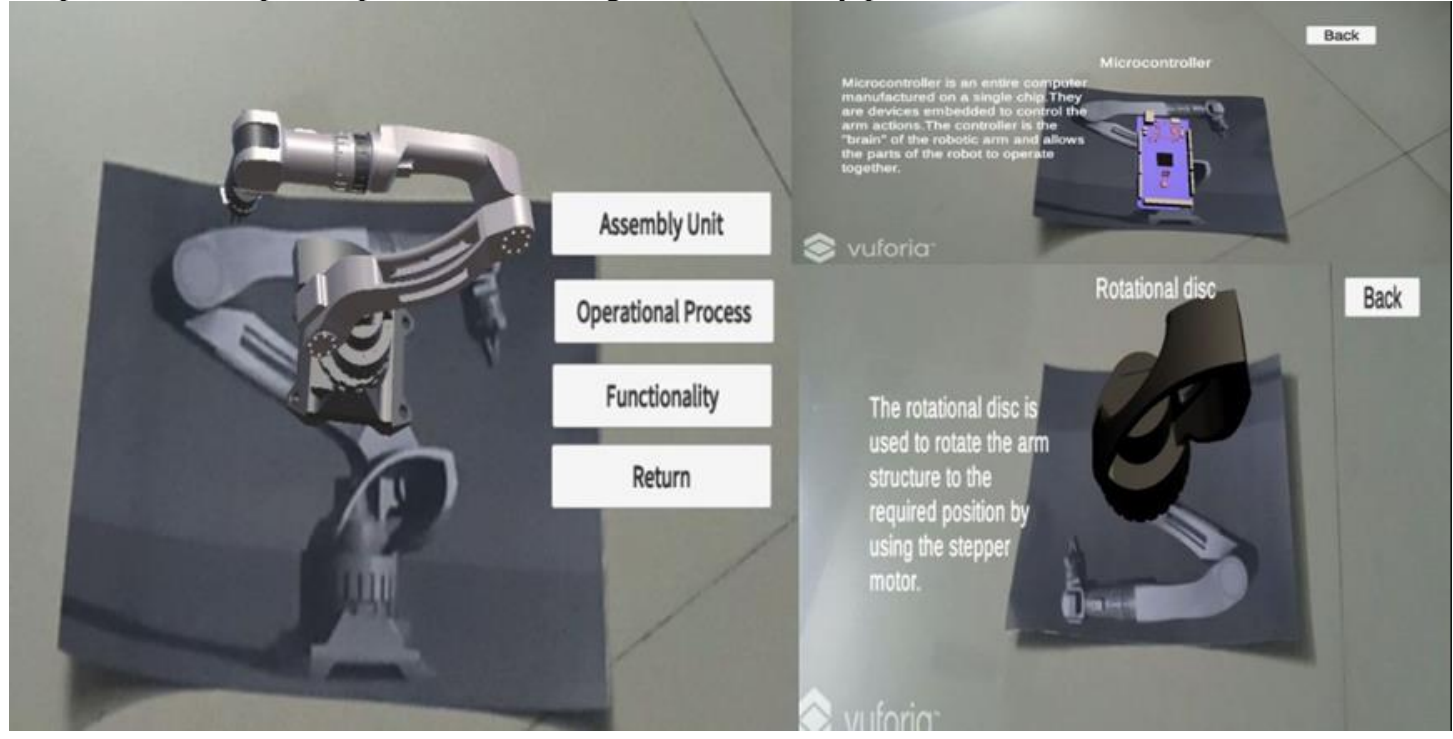

Figure 6 Mobile AR Application

Figure 6 shows the screenshot of the developed AR application(Marker-based Augmented Reality)through the mobile phone. Therobotic arm, its working, functions and operations in the AR application is shown. Similar work is also carried out for concepts from physics and biology The image target AR experience can provide students, the real-time feel of using them. Here 3D objects get augmented in the real environment which gives an immersive experience for students which gives them in-depth understanding.

\section{Discussion}

This work can help rural school students as well as faculties to overcome most of their issues. From the feedback of some school students, we found that the application will have a very good social impact. This app provides experimental learning and the major advantage is that the student can learn from this app offline and also, they can learn from it anywhere, anytime from the device. They can also revise the classes any number of times as required by the student. They can get proper education even during the occurrence of any pandemic situation like COVID 19. In such fatal situations, it is not possible for conducting offline live classes. For online classes, they should ensure that the students have proper internet learning facilities as their home

\section{Conclusion}

Smart AR learning would greatly benefit the students to develop "do by learning" skill. Students can efficiently learn the concepts and procedures within a very short time and easily understand the application as well the schools need not have all the equipment physically present, rather they can visualize it using our application.

\section{References}

1. Yang Kuang, XiaoMei. The Feasibility Study of Augmented Reality Technology in Early 
2. Childhood Education. The 14th International Conference on Computer Science \&

3. Education (ICCSE 2019), Toronto, Canada,2019

4. Jia Zhang, Kuo-En Chang. Applying Augmented Reality to Improve the Outcomes of 5. Procedural Knowledge Acquisition.IEEE 19th International Conference on Advances 6.Learning Technologies (ICALT). IEEE DOI 10.1109/ICALT.2019.00105, 2019.

7. Patrício J M, Costa M C, Manso A. A Gamified Mobile Augmented Reality System for 8. the teaching of astronomical concepts. 14th Iberian Conference on Information Systems 9. and Technologies (CISTI),Portugal,2019. ISBN: 978-989-98434-9-3.

10. Su Cai, Xu Wang, MengnanGan, Shengquan Yu. Simulation Teaching in 3D Augmented

11. Reality Environment. 978-0-7695-4826-5/, 2012 IEEE DOI 10.1109/IIAI-AAI.2012.25.

12. Iuri Victor Costa, ViniciusFavachoQueiroz, Brunelli Pinto Miranda. A Card Based

13. Interaction to Design Visualizations in Augmented Reality Environments.978-1-7281-

14. 2850-4/19,2019 IEEE DOI 10.1109/IV -2.2019.00019

15. Kaufmann H and Meyer B. Simulating Educational Physical Experiments in Augmented

16. Reality. ACM, p. 3, 2018.

17. Somasundaram, T. S., Kiruthika, U., Gowsalya, M., Hemalatha, A., \& Philips, A. (2015, May). Determination of competency of programmers by classification and ranking using AHP. In 2015 IEEE International Conference on Electro/Information Technology (EIT) (pp. 194-200). IEEE.

18. Raja, S. Kanaga Suba, and T. Jebarajan. "Reliable and secured data transmission in wireless body area networks (WBAN)." European Journal of Scientific Research 82, no. 2 (2012): 173-184.

19. Hindustan Times [Internet] Available from

20. https://www.hindustantimes.com/education/all-government-schools-in-up-to-have-library-bymarch-2020/story-pIpsrN2Unar9sRTK3pUEVN.html

21. Sampathkumar, A., Murugan, S., Sivaram, M., Sharma, V., Venkatachalam, K. and Kalimuthu, M., 2020. Advanced Energy Management System for Smart City Application Using the IoT. In Internet of Things in Smart Technologies for Sustainable Urban Development (pp. 185-194). Springer, Cham.

22. Sampathkumar, A., Murugan, S., Rastogi, R., Mishra, M.K., Malathy, S. and Manikandan, R., 2020. Energy Efficient ACPI and JEHDO Mechanism for IoT Device Energy Management in Healthcare. In Internet of Things in Smart Technologies for Sustainable Urban Development (pp. 131-140). Springer, Cham. 